NASA TECHNICAL NOTE

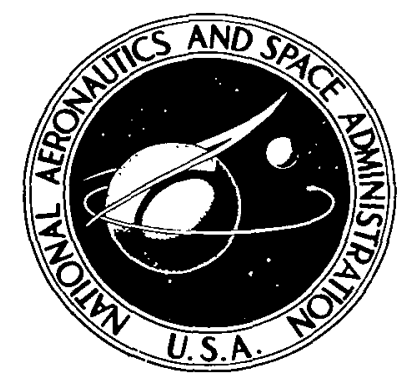

NASA IN D-6074

IOAN C. 1

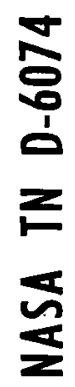
AFWL (WIOL) KIRTLLAND AFB, N N

\title{
EFFECTS OF CORRELATION ON THE
} STRUCTURE OF IMPURITY BANDS

by Gust Bambakidis

Lewis Research Center Cleveland, Obio 44135

NATIONAL AERONAUTICS AND SPACE ADMINISTRATION - WASHINGTON, D. C. NOVEMBER 1970 
1. Report No.

NASA TN D-6074

4. Title and Subtitle EFFECTS OF CORRELATION ON THE STRUCTURE OF IMPURITY BANDS

7. Author(s)

Gust Bambakidis

9. Performing Organization Name and Address

Lewis Research Center

National Aeronautics and Space Administration

Cleveland, Ohio 44135

12. Sponsoring Agency Name and Address

National Aeronautics and Space Administration

Washington, D. C. 20546

15. Supplementary Notes

16. Abstract

The effects of electron-electron interaction on the electronic density of states has been considered for a dilute system of scattering centers distributed randomly in an electron gas of moderate density. Using as the starting point an independent-electron $t$-matrix method, a modified $t$-matrix in the presence of the electron-electron interaction is obtained. To illustrate the effects of correlation, the formalism has been applied to the simple case of a linear chain of attractive $\delta$-function potentials distributed randomly. The electrons are assumed to interact by means of a repulsive $\delta$-function potential. The density of states in the impurity band is evaluated and compared with the results for independent electrons. It is found that the inclusion of electron-electron interaction produces a significant broadening and downward energy shift of the impurity band.

17. Key Words (Sugkested by Author(s))

Impurity states Electron correlation
18. Distribution Statement

Unclassified - unlimited
19. Security Classif. (of this report) Unclassified
20. Security Classif. (of this page) Unclassified
21. No. of Pages 16
22. Price* $\$ 3.00$

*For sale by the Clearinghouse for Federal Scientific and Technical Information Springfield, Virginia 22151 


\title{
EFFECTS OF CORRELATION ON THE STRUCTURE OF IMPURITY BANDS
}

\author{
by Gust Bambakidis
}

\author{
Lewis Research Center
}

\section{SUMMARY}

The effects of electron-electron interaction on the electronic density of states has been considered for a dilute system of scattering centers distributed randomly in an electron gas of moderate density. Using as the starting point an independent-electron $\mathrm{t}$-matrix method, a modified $\mathrm{t}$-matrix in the presence of the electron-electron interaction is obtained. To illustrate the effects of correlation, the formalism has been applied to the simple case of a linear chain of attractive $\delta$-function potentials distributed randomly. The electrons are assumed to interact by means of a repulsive $\delta$-function potential. The density of states in the impurity band is evaluated and compared with the results for independent electrons. It is found that the inclusion of electron-electron interaction produces a significant broadening and downward energy shift of the impurity band.

\section{INTRODUCTION}

Many papers dealing with the electronic structure of disordered systems have appeared. Because of the difficulty of the subject, for which there exists no general one-electron theory, these treatments have, for the most part, considered the electrons as noninteracting. Wolff (ref. 1) has treated the modification of a free-electron band in the presence of both the electron-impurity and the electron-electron interactions. However he considers the case of very high impurity and electron densities (relative to the effective Bohr radius of the impurity), so that no localized states arising from singleimpurity bound states can occur. The transport properties of interacting electrons in a system of random impurities have also been treated extensively by Langer (refs. 2 to 5 ), by Betbeder-Matibet and Nozières (ref. 6), and more recently by Sigel and Argyres (ref. 7) and by Sigel (ref. 8). Here we describe a calculation of the effect of particleparticle interaction on the density of single-particle states in the band which arises from a bound state of an isolated impurity. In order to describe the impurity band without 
many-particle effects, the $t$-matrix approach of des Cloizeaux (ref. 9) is used. This approach is based on infinite-order perturbation theory, and, consequently, many-body effects can be included in a straightforward way using standard propagator formalism (refs. 10 and 11).

\section{CALCULATION}

The Hamiltonian for a system of fixed-volume $\Omega$ at $\mathrm{T} \approx 0$ containing $\mathrm{N}_{\mathrm{e}}$ particles interacting with $\mathrm{N}_{\mathrm{a}}$ impurities distributed at random is written. The impurities are assumed to be infinitely massive. Then

$$
H=\sum_{i=1}^{N_{e}} T_{i}+\sum_{i=1}^{N_{e}} v_{a}\left(\underline{r}_{i}\right)+\frac{1}{2} \sum_{\substack{i, j=1 \\ i \neq j}}^{N_{e}} v_{e}\left(\underline{r}_{i}-\underline{r}_{j}\right)-\sum_{i=1}^{N_{e}} v_{o a}-\frac{1}{2} \sum_{i=1}^{N_{e}} v_{o e}
$$

where

$$
\mathrm{T}_{\mathrm{i}}=-\frac{\hbar^{2}}{2 \mathrm{~m}_{\mathrm{e}}} \nabla_{\mathrm{i}}^{2}
$$

is the kinetic-energy operator for the $i^{\text {th }}$ particle and

$$
\mathrm{v}_{\mathrm{a}}\left(\underline{\mathrm{r}}_{\mathrm{i}}\right)=\sum_{\alpha=1}^{\mathrm{N}_{\mathrm{a}}} \mathrm{v}_{\mathrm{a}}\left(\underline{\mathrm{r}}_{\mathrm{i}}-\underline{\mathrm{R}}_{\alpha}\right)
$$

is the potential energy of the $i^{\text {th }}$ particle due to the interactions $v_{a}\left(r_{i}-\underline{R}_{\alpha}\right)$ between the $i^{\text {th }}$ particle and the impurities distributed among the sites $\underline{R}_{\alpha}$. All physical quantities are to be averaged over the (random) distribution of the $\underline{R}_{\alpha}$. We consider the system as being "neutral;" that is, assume that there are potential sources which compensate for the potential energy of a particle in the average potential fields of the impurities $V_{\text {oa }}$ and of the particle gas $V_{\text {oe }}$. The perturbation calculation is carried out by using the second-quantized form of $\mathrm{H}$ : 


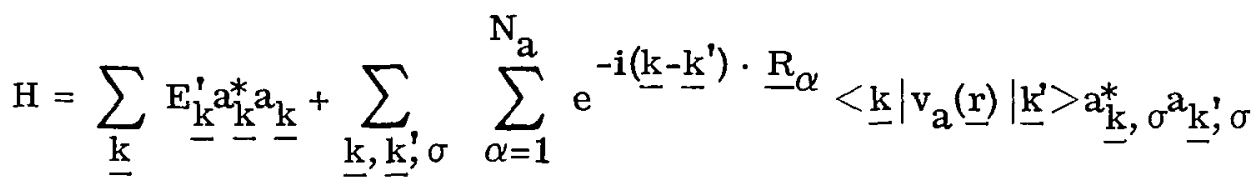

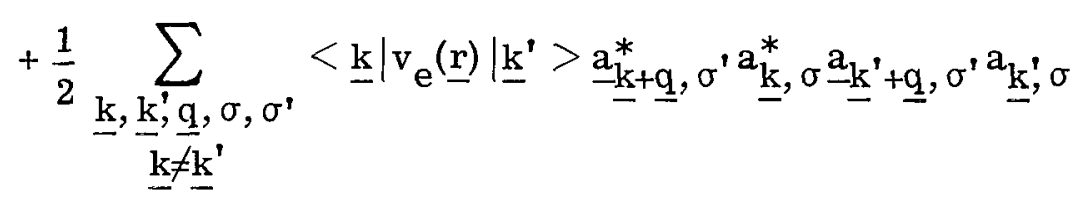

where

$$
\mathrm{E}_{\underline{\mathrm{k}}}^{\prime}=\mathrm{E}_{\underline{\mathrm{k}}}-\mathrm{v}_{\mathrm{oa}}
$$

and

$$
\mathrm{E}_{\underline{\mathrm{k}}}=\frac{\hbar^{2} \mathrm{k}^{2}}{2 \mathrm{~m}_{\mathrm{e}}}
$$

The effect of the constant term $-V_{\text {oe }}$ in equation (1) is to remove the diagonal elements $\left\langle\underline{\mathrm{k}}\left|\mathrm{v}_{\mathrm{e}}\right| \underline{\mathrm{k}}\right\rangle$ from the particle-particle portion of $\mathrm{H}$. Hence, $\underline{\mathrm{k}} \neq \underline{\mathrm{k}} \underline{\mathrm{j}}^{\prime}$ in equation (1'). The constant term $-\mathrm{V}_{\mathrm{oa}}$ in equation (1) simply shifts the scale of single-particle energies:

$$
\mathrm{E}=\mathrm{E}^{\prime}+\mathrm{V}_{\mathrm{Oa}}=\mathrm{E}^{\prime}+\mathrm{N}_{\mathrm{a}}<\underline{\mathrm{k}}\left|\mathrm{v}_{\mathrm{a}}\right| \underline{\mathrm{k}}>
$$

where $E^{\prime}$ is the energy referred to the unshifted energy scale. (However, when $v_{a}$ is long-ranged (e.g., Coulombic), this energy shift is divergent and $-\mathrm{V}_{\mathrm{oa}}$ has to be included implicitly by removing the diagonal elements from the particle-impurity interaction.)

We wish to obtain the single-particle propagator $\mathrm{G}(\mathrm{k}, \omega)$ for the system. The density of single-particle states will then be given by

$$
\begin{aligned}
\mathrm{D}(\mathrm{E}) & =\frac{1}{\pi} \operatorname{Im} \sum_{\underline{\mathrm{k}}} \mathrm{G}(\underline{\mathrm{k}}, \mathrm{E}) & & \mathrm{E} \leq \mathrm{E}_{\mathrm{F}} \\
& =-\frac{1}{\pi} \operatorname{Im} \sum_{\underline{\mathrm{k}}} \mathrm{G}(\underline{\mathrm{k}}, \mathrm{E}) & & \mathrm{E}>\mathrm{E}_{\mathrm{F}}
\end{aligned}
$$


(Unless otherwise indicated, the index $\underline{\mathrm{k}}$ includes implicitly the spin quantum number $\sigma= \pm 1 / 2$.) First, consider the case without particle-particle interaction $\left(v_{e}=0\right)$. The Hamiltonian becomes a sum of $\mathrm{N}_{e}$ independent one-particle Hamiltonians $\mathrm{H}_{\mathbf{i}}=\mathrm{T}_{\mathrm{i}}+$ $V_{a}\left(\underline{r}_{i}\right)$, and it is sufficient to obtain $G(k, \omega)$ for the system of one particle plus impurities. The propagator can be written as a summation of an infinite series of multiple-scattering

diagrams involving the free-particle propagator $G_{0}(\underline{k}, \omega)$. Denoting $G(\vec{k}, \omega)$ by $\vec{k}, \omega \|$ and $\mathrm{G}_{\mathrm{o}}(\overrightarrow{\mathrm{k}}, \omega)$ by $\overrightarrow{\mathrm{k}}, \omega \uparrow$ yields

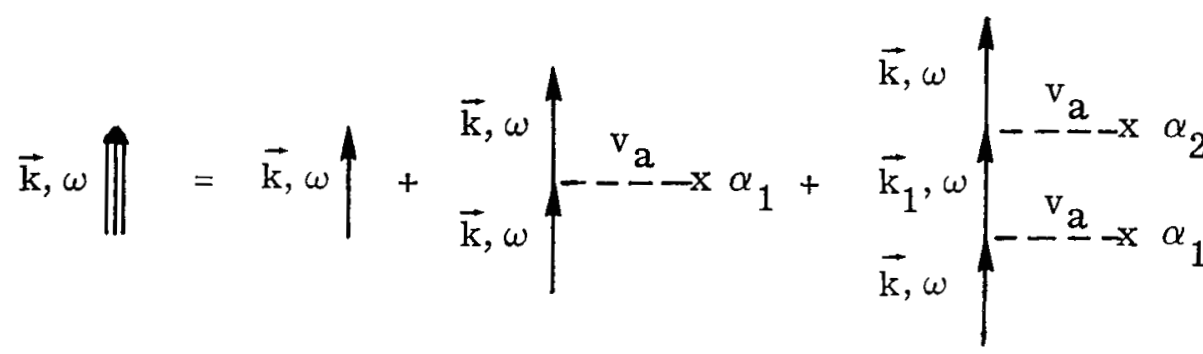

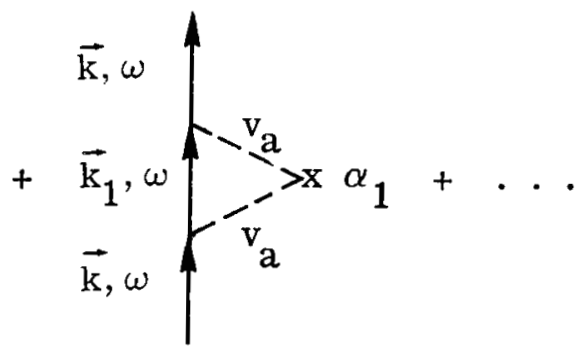

Next, the potential $v_{a}$ is replaced by the particle-impurity $t$-matrix $t_{a}(\omega)$, which is the sum of all multiple scatterings of the particle by the impurity $\alpha$ :

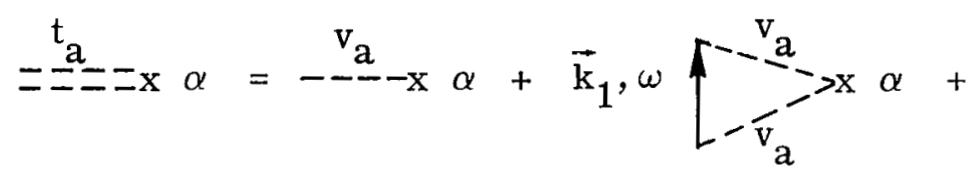

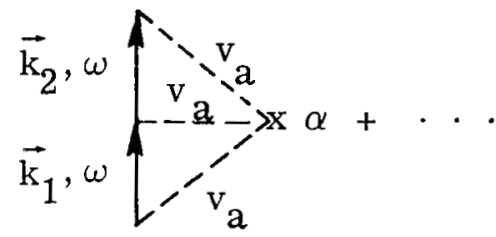


In reference 9 , des Cloizeaux has introduced the notion of articulation points in these diagrams. By definition, an articulation point is associated with an interaction of the particle with the impurity $\alpha$ if all scattering centers encountered by the particle before this interaction $\beta(\beta \neq \alpha)$ are different from all scattering centers encountered after this interaction $\gamma(\gamma \neq \alpha)$. As a consequence of the averaging process, each diagram can then be reduced to a product of irreducible parts by cutting the propagator lines connected to each articulation point in the diagram. Propagation between articulation points can be described by a "self-propagator"' $\mathrm{s}(\underline{\mathrm{k}}, \omega)$, with matrix element

$$
\left\langle\overrightarrow{\mathrm{k}}_{2}|\mathrm{~s}(\overrightarrow{\mathrm{k}}, \omega)| \overrightarrow{\mathrm{k}}_{1}\right\rangle \equiv \begin{aligned}
& \overrightarrow{\mathrm{k}}_{2} \\
& \overrightarrow{\mathrm{k}}, \omega \\
& \overrightarrow{\mathrm{k}}_{1}
\end{aligned}
$$

Upon associating the operator $\rho_{\mathrm{a}} \mathrm{t}_{\mathrm{a}}(\omega) \equiv \mathrm{N}_{\mathrm{a}} / \Omega \mathrm{t}_{\mathrm{a}}(\omega)$ with each articulation point denoted by $O$, the diagrammatic expansion of $G(\underline{k}, \omega)$ can be redrawn as

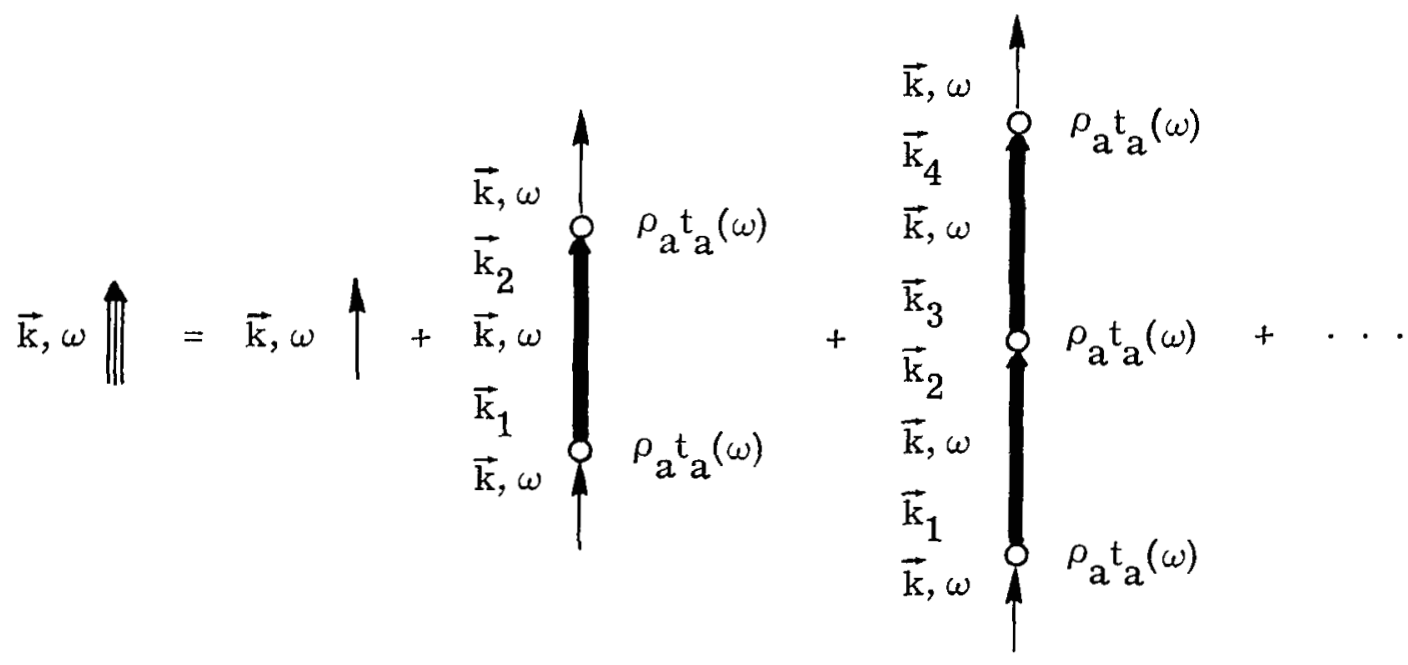

leading to

$$
\left.\mathrm{G}(\underline{\mathrm{k}}, \omega)=\mathrm{G}_{\mathrm{o}} \underline{(\mathrm{k}}, \omega\right)+\mathrm{G}_{\mathrm{o}}^{2}(\underline{\mathrm{k}}, \omega)<\underline{\mathrm{k}}\left|\rho_{\mathrm{a}} \mathrm{t}_{\mathrm{a}}(\omega)\left[1-\rho_{\mathrm{a}} \mathrm{S}(\underline{\mathrm{k}}, \omega) \mathrm{t}_{\mathrm{a}}(\omega)\right]^{-1}\right| \underline{\mathrm{k}}>
$$


The self-propagator $S$ can, in turn, be expanded in powers of the impurity density with the leading term, of order $\rho_{a}^{2}$, containing the contribution of scattering by pairs of impurities.

In considering the effects of electron-electron interaction, we are particularly interested in the broadening and shift of the impurity band at moderate to high electron densities. Consequently, the effect on a particle of a single impurity is examined in the presence of all the other particles. This approach leads to a modification of the particleimpurity $t$-matrix while neglecting correlation effects on the scattering of the particle between different impurities. For each "skeleton" particle-particle scattering diagram contributing to the propagator in the absence of this impurity, the corresponding diagram in its presence is obtained by replacing everywhere the free-particle propagator with the "dressed" propagator appropriate to the system of a particle plus one impurity:

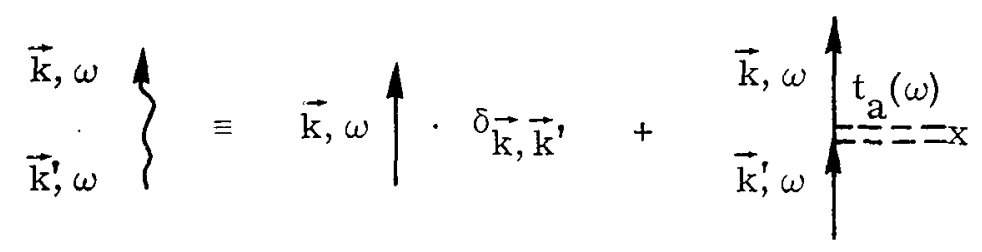

Consider irreducible diagrams: that is, those which cannot be cut into two others by cutting a dressed propagator line. We denote by $\Sigma\left(\underline{k}, \mathrm{k}^{\prime}, \omega\right)$ the sum of all irreducible diagrams, taking the particle from state $\underline{\mathrm{k}}^{\prime}$ to state $\underline{\mathrm{k}}$, with energy $\hbar \omega$ :

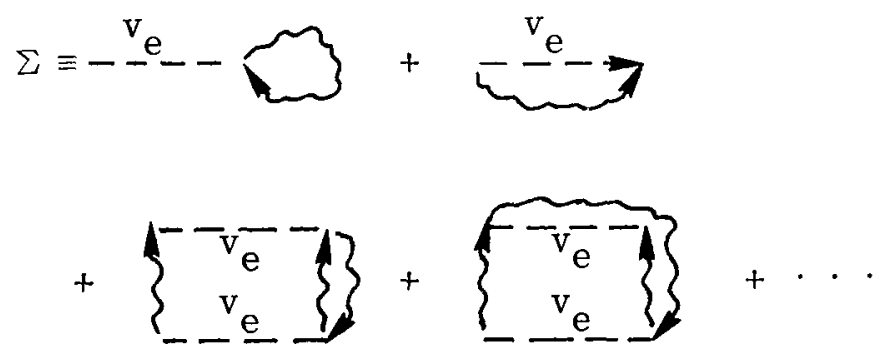

Because the presence of the impurity disrupts the homogeneity of the particle gas, the term $\Sigma(\underline{k}, \underline{k}, \omega)$ differs from the usual self-energy in that it is nondiagonal. For this

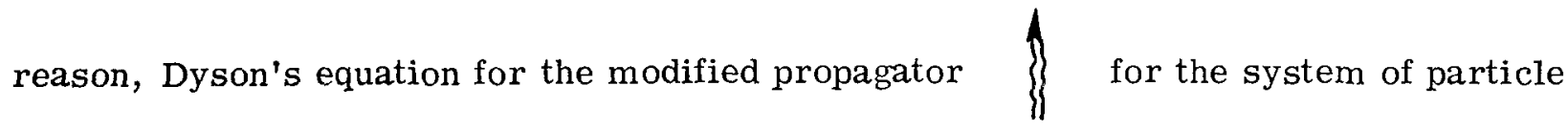
gas plus one impurity cannot be summed into closed form. We can write only 


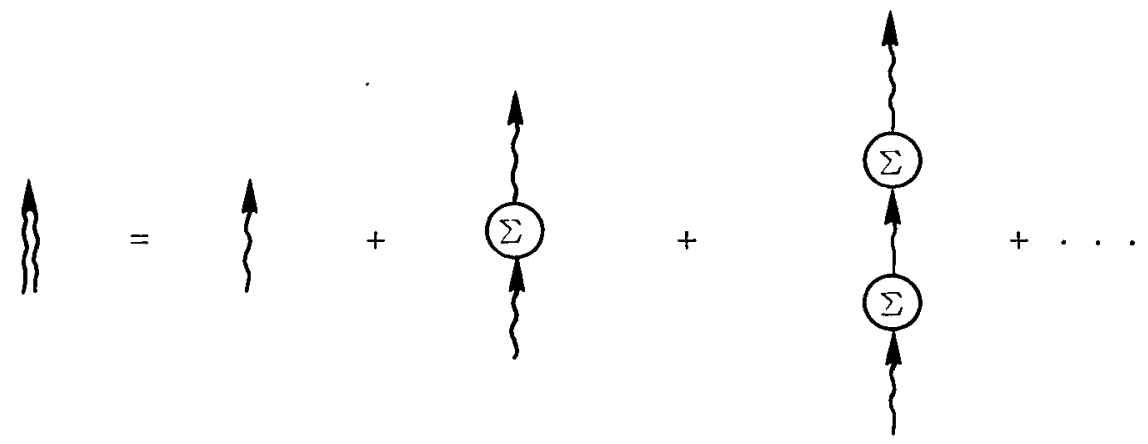

The effective $t$ matrix $t_{a}^{\text {eff }}$ is defined in terms of $\}$ by

$$
\begin{aligned}
& \overrightarrow{\mathrm{k}}, \omega \uparrow \mathrm{t}_{\mathrm{a}}^{\mathrm{eff}(\omega)} \overrightarrow{\mathrm{k}}, \omega \\
& \overrightarrow{\mathrm{k}}, \omega
\end{aligned}
$$

where $\vec{k}, \omega \|$ is the propagator $G_{e}(\vec{k}, \omega)$ in the presence of the particle gas only.

To obtain $t_{a}^{\text {eff }}$ we must know $\Sigma$. To make the situation tractable let us in fact approximate $\Sigma$ by $\delta_{\underline{k}, k^{\prime}} \Sigma_{e}(\underline{k}, \omega)$, where $\Sigma_{e}$ is the (diagonal) self-energy without the impurity.

The expression for $\{$ then becomes 


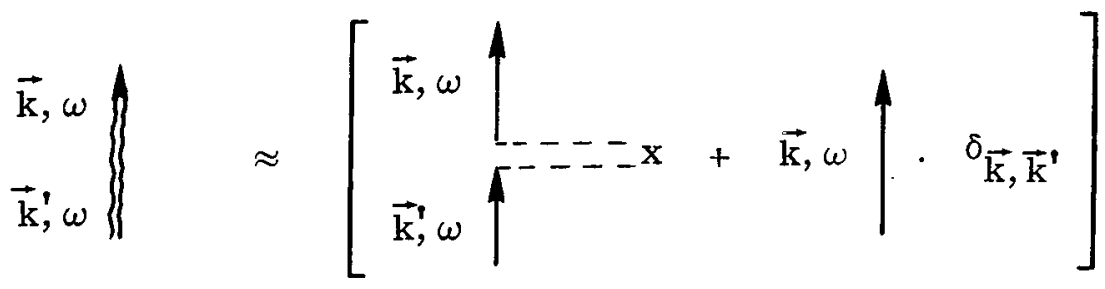

$\left[\begin{array}{ll}\overrightarrow{\mathrm{k}}, \omega \\ \overrightarrow{\mathrm{k}}_{1}, \omega \\ \overrightarrow{\mathrm{k}}_{1}^{\prime}, \omega\end{array}\right.$

This can be resummed as

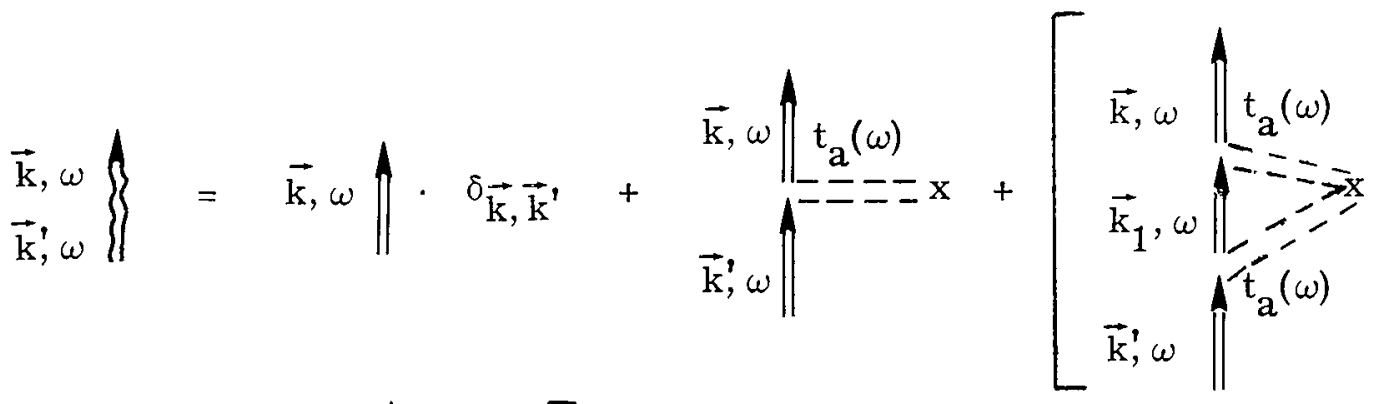

$$
\begin{aligned}
& \overrightarrow{\mathrm{k}}_{1}^{\prime}, \omega
\end{aligned}
$$


where

$$
\overrightarrow{\mathrm{k}}, \omega \|=\overrightarrow{\mathrm{k}}, \omega \mid \overrightarrow{\mathrm{k}}, \omega)+\cdots
$$

Let us now consider the simple case of a disordered linear chain of attractive $\delta$ function potentials. In this case,

$$
v_{a}\left(x-x_{\alpha}\right)=-v_{o}^{a} \delta\left(x-x_{\alpha}\right)
$$

and the $t$-matrix is

$$
<\mathrm{k}\left|\mathrm{t}_{\mathrm{a}}(\omega)\right| \mathrm{k}^{\prime}>=\frac{\frac{-\mathrm{v}_{\mathrm{o}}^{\mathrm{a}}}{\Omega}}{1-\frac{\mathrm{iv}_{\mathrm{o}}^{\mathrm{a}}}{2(\omega+\mathrm{io})^{1 / 2}}} \quad \omega<0
$$

in atomic units $\left(\hbar=1, \mathrm{~m}_{\mathrm{e}}=1 / 2, \mathrm{e}^{2} / 4 \pi \epsilon_{\mathrm{o}}=2\right)$. This has a singularity at the bound-state energy $E_{b}=-\left(v_{0}^{a} / 2\right)^{2}$ of a particle in the field of one impurity. The fact that the matrix element of $t_{a}$ is independent of initial and final momenta simplifies matters considerably since equation (7') for $\quad \mathrm{G}_{\mathrm{a}-\mathrm{e}}(\overrightarrow{\mathrm{k}}, \overrightarrow{\mathrm{k}}, \omega) \equiv \overrightarrow{\mathrm{k}}, \omega$ particles plus one impurity, can be summed into closed form: 


$$
\begin{aligned}
& G_{\left.a-e^{(k, k}, \omega\right)}=G_{e}(k, \omega) \cdot \delta_{k, k^{\prime}}+G_{e}\left(k^{\prime}, \omega\right) t_{a}(\omega) G_{e}(k, \omega) \\
& +G_{e}\left(k^{\prime}, \omega\right) t_{a}(\omega)\left\{\sum_{k_{1}}\left[G_{e}\left(k_{1}, \omega\right)-G_{o}\left(k_{1}, \omega\right)\right]\right\} t_{a}(\omega) G_{e}(k, \omega) \\
& +\cdot \cdot \\
& =G_{e}(k, \omega) \cdot \delta_{k, k^{\prime}}+G_{e}(k, \omega)\left\{\sum_{n=0}^{\infty} t_{a}^{n+1}(\omega) g^{n}(\omega)\right\} G_{e}(k, \omega) \\
& =G_{e}(k, \omega) \cdot \delta_{k, k^{\prime}}+G_{e}\left(k^{\prime}, \omega\right) t_{a}(\omega)\left[1-t_{a}(\omega) S(\omega)\right]^{-1} G_{e}(k, \omega),
\end{aligned}
$$

where

$$
\begin{aligned}
& S(\omega)=\sum_{\mathrm{k}_{1}}\left[\mathrm{G}_{\mathrm{e}}\left(\mathrm{k}_{1}, \omega\right)-\mathrm{G}_{\mathrm{o}}\left(\mathrm{k}_{1}, \omega\right)\right] \\
& =\sum_{\mathrm{k}_{1}}\left[\frac{\mathrm{G}_{\mathrm{o}}\left(\mathrm{k}_{1}, \omega\right) \Sigma_{\mathrm{e}}\left(\mathrm{k}_{1}, \omega\right) \mathrm{G}_{\mathrm{o}}\left(\mathrm{k}_{1}, \omega\right)}{1-\Sigma_{\mathrm{e}}\left(\mathrm{k}_{1}, \omega\right) \mathrm{G}_{\mathrm{o}}\left(\mathrm{k}_{1}, \omega\right)}\right]
\end{aligned}
$$

and $t_{a}(\omega)$ has been written for $\left\langle\mathrm{k}\left|\mathrm{t}_{\mathrm{a}}(\omega)\right| \mathrm{k}^{\prime}\right\rangle$. From equation (6) we see that

$$
t_{a}^{e f f}(\omega)=\left[1-\Sigma_{e}\left(k^{\prime}, \omega\right) G_{o}\left(k^{\prime}, \omega\right)\right]^{-1} t_{a}(\omega)\left[1-t_{a}(\omega) \mathcal{S}(\omega)\right]-1 \cdot\left[1-\Sigma_{e}(k, \omega) G_{o}(k, \omega)\right]^{-1},
$$

and is dependent upon $\mathrm{k}^{\prime}$ and $\mathrm{k}$. To facilitate comparison with the results using the bare t-matrix, this dependence is ignored:

$$
\mathrm{t}_{\mathrm{a}}^{\mathrm{eff}}(\omega) \approx \mathrm{t}_{\mathrm{a}}(\omega)\left[1-\mathrm{t}_{\mathrm{a}}(\omega) \mathcal{S}(\omega)\right]^{-1}
$$

The quantity $S(\omega)$ has been evaluated when the particles or "electrons" interact by means of a repulsive $\delta$-function: 


$$
v_{e}\left(x_{i}-x_{j}\right)=v_{o}^{e} \delta\left(x_{i}-x_{j}\right)
$$

The self-energy was calculated in the ladder approximation to lowest order in the particle-particle $t$-matrix $t_{e}(\underline{q}, \zeta)$ :

$$
\begin{aligned}
& \Sigma_{\mathrm{e}}(\overrightarrow{\mathrm{k}}, \omega) \approx\left[=\frac{\mathrm{t}_{\mathrm{e}}(\overrightarrow{\mathrm{q}}, \zeta)}{=}==\bigcirc \overrightarrow{\mathrm{q}}-\overrightarrow{\mathrm{k}}, \zeta-\omega-\cdots \overrightarrow{\mathrm{v}}-\overrightarrow{\mathrm{k}}, \zeta-\omega\right] \\
& +\underset{\overrightarrow{\mathrm{q}}-\overrightarrow{\mathrm{k}}, \zeta-\omega}{=\frac{\mathrm{t}_{\mathrm{e}}(\overrightarrow{\mathrm{q}}, \zeta)}{=}=ニ=}=-\frac{\mathrm{i}}{2 \pi} \cdot 2 \sum_{\overrightarrow{\mathrm{q}}} \int_{-\infty}^{\infty} \mathrm{d} \zeta\left[\left\langle\overrightarrow{\mathrm{k}}\left|\mathrm{t}_{\mathrm{e}}(\overrightarrow{\mathrm{q}}, \zeta)\right| \overrightarrow{\mathrm{k}}\right\rangle-\left\langle\overrightarrow{\mathrm{k}}\left|\mathrm{v}_{\mathrm{e}}\right| \overrightarrow{\mathrm{k}}\right\rangle\right] \mathrm{G}_{\mathrm{o}}(\overrightarrow{\mathrm{q}}-\overrightarrow{\mathrm{k}}, \zeta-\omega) \\
& +\frac{i}{2 \pi} \sum_{\vec{q}} \int_{-\infty}^{\infty} d \zeta<\vec{q}-\vec{k}\left|t_{e}(\vec{q}, \zeta)\right| \vec{k}>G_{0}(\vec{q}-\vec{k}, \zeta-\omega)
\end{aligned}
$$

In this case $<\underline{\mathbf{k}}\left|\mathrm{t}_{\mathrm{e}}(\underline{\mathrm{q}}, \zeta)\right| \underline{\mathrm{k}^{\prime}}>$ depends only on $\mathrm{q}$ and $\zeta$ and can be obtained in closed form as

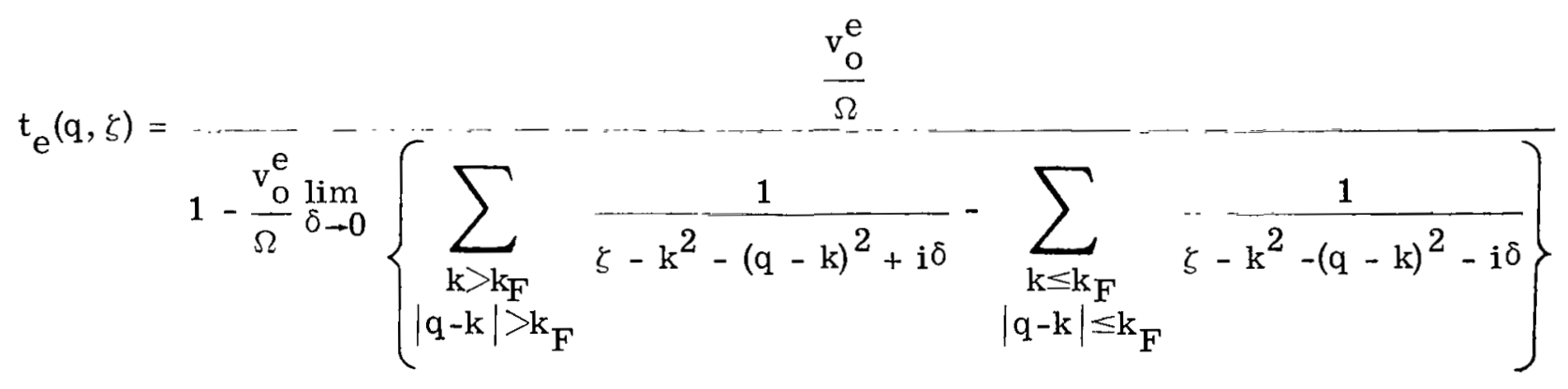

\section{DISCUSSION}

The expression which results when the free-particle propagator $G_{\mathrm{o}}(\mathrm{k}, \omega)$ appearing in equation (5) is replaced by $G_{e}(\underline{k}, \omega)$ and the bare particle-impurity $t$-matrix $t_{a}$ by $\mathrm{t}_{\mathrm{a}}^{\mathrm{eff}}$ is 


$$
\mathrm{G}(\underline{k}, \omega)=G_{e}(\underline{k}, \omega)+G_{e}^{2}(\underline{k}, \omega)<\underline{k}\left|\rho_{\mathrm{a}} \mathrm{t}_{\mathrm{a}}^{\mathrm{eff}}(\omega)\left[1-\rho_{\mathrm{a}} \mathrm{S}(\underline{\mathrm{k}}, \omega) \mathrm{t}_{\mathrm{a}}^{\mathrm{eff}}(\omega)\right]^{-1}\right| \underline{\mathrm{k}}>.
$$

This equation reduces to the correct limit when either $v_{a}$ or $v_{e}$ vanishes. Upon computing $G_{e}$ from equation (8) and $t_{a}^{\text {eff }}$ from equation (11), the resulting density of states (per unit length) obtained for negative energies is shown in figure 1, together with the result for independent electrons. In both cases we have used $\mathrm{v}_{\mathrm{o}}^{\mathrm{a}}=2 \mathrm{Ry}-\mathrm{a}_{0}$ and $\rho_{\mathrm{a}}=$ $1 / 3 \mathrm{a}_{\mathrm{o}}^{-1}$, while in the former case we have taken $\mathrm{v}_{\mathrm{o}}^{\mathrm{e}}=\mathrm{v}_{\mathrm{o}}^{\mathrm{a}}$ and $\rho_{\mathrm{e}}^{\mathrm{o}}=1 \mathrm{a}_{\mathrm{o}}^{-1}$. The lowest order expansion in $\rho_{a}$ of the self-propagator was used. (The self-propagator $\mathbf{S}(\underline{k}, \omega)$ consists of the sum of two terms $\mathrm{A}(\omega)+\mathrm{B}(\underline{\mathrm{k}}, \omega)$. To lowest order in $\rho_{\mathbf{a}}$, only the first term has been retained as giving, in this particular case, the dominant contribution to $\mathrm{D}(\mathrm{E})$ in the neighborhood of the maximum (see ref. 9).) Also shown is a machine calculation for independent electrons, reported in reference 6 . We see that the effect of particle-particle interaction on the impurity band is to displace it to lower energies as well as to broaden it. For the parameter values used here the full width at half maximum with particle interaction ( $0.42 \mathrm{Ry})$ is comparable to that occurring in the machine cal-

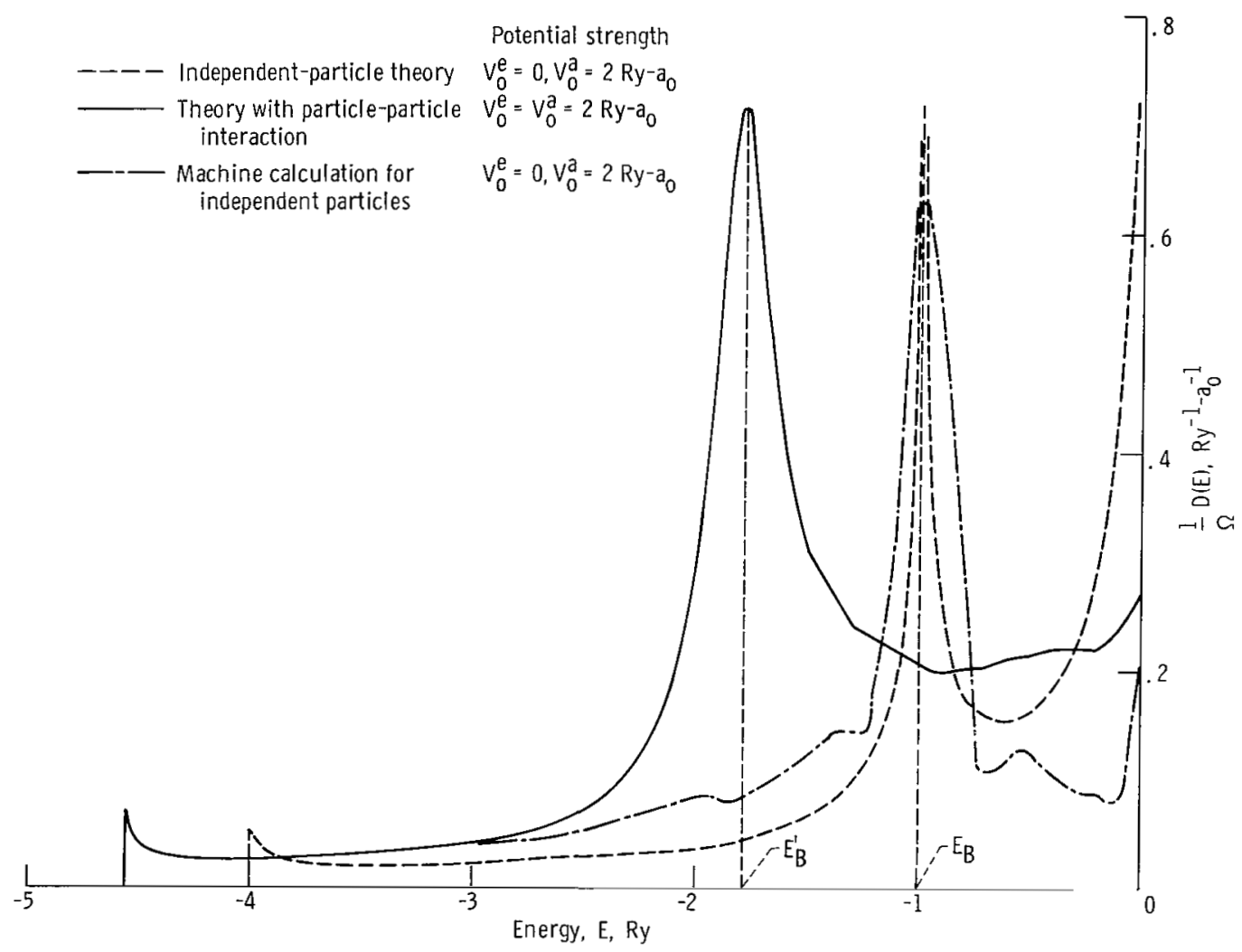

Figure 1. - Single-particle density of states, per unit iength, in the impurity band for a linear chain of random scatterers. Scatterer density, $\rho_{a}=1 / 3 a_{0}^{-1}$; particle density, $\rho_{e}=1 a_{0}^{-1}$. 
culation for independent particles $(\sim 0.30 \mathrm{Ry})$. To estimate the shift of the impurity band with respect to the Fermi level, the following approximate relation for a filled band $\left(\rho_{\mathrm{e}}>2 \rho_{\mathrm{a}}\right)$ was used in obtaining $\mathrm{E}_{\mathrm{F}}$ :

$$
\int_{-\infty}^{E_{F}} \frac{1}{\Omega}\left[D(E)-D_{e}(E)\right] d E \sim 2 \rho_{a}
$$

or

$$
\int_{-\infty}^{E} \frac{1}{\Omega} D_{e}(E) d E \sim \rho_{e}-2 \rho_{a}
$$

where $D_{e}(E)$ is the contribution to the state density from the first term in equation (15). Equation (16) is a good approximation in the independent-particle theory, for which $\int_{-\infty}^{0}(1 / \Omega) \mathrm{D}(\mathrm{E}) \mathrm{dE} \approx 2 \rho_{\mathrm{a}}$. In general, we would expect it to be applicable in the tightbinding limit ( $\rho_{\mathrm{a}}$ small relative to the bound-state radius of an isolated impurity) for $\mathrm{v}_{\mathrm{e}}$ small compared to $\mathrm{v}_{\mathrm{a}}$. Using equation $\left(16^{\prime}\right)$ we obtain $\mathrm{E}_{\mathrm{F}} \sim 0.27 \mathrm{Ry}, \mathrm{E}_{\mathrm{F}}^{\prime} \sim-0.03 \mathrm{Ry}$, so that the shift is

$$
\left(E_{B}^{\prime}-E_{F}^{\prime}\right)-\left(E_{B}-E_{F}\right) \sim-0.49 R y
$$

The integrated state density up to $E=E_{F}^{\prime}$ is

$$
\int_{-\infty}^{E_{F}^{\prime}} \frac{1}{\Omega} \mathrm{D}(\mathrm{E}) \mathrm{dE}=0.737 \frac{\text { states }}{\mathrm{a}_{\mathrm{o}}}<\rho_{\mathrm{e}}
$$

Thus equation (16) underestimates the shifted Fermi level in this case $\left(v_{e}=v_{a}\right)$.

The above results indicate that while broadening of the impurity and conduction bands due to interparticle interaction increases their overlap, there is a net downward shift of the center of the impurity band with respect to the Fermi level.

In three dimensions, the effect of particle-particle interaction on an impurity band should be more pronounced than in one dimension. In three dimensions, strong screening of an impurity at high electron densities can cause it to ionize completely, whereas a one-dimensional potential well always possesses at least one bound state. We believe that the overall results of this calculation are reasonable. In treating a more realistic (e.g., three dimensional) case, however, the computational problem becomes rather 
formidable. An accurate determination of $\Sigma_{\mathrm{e}}(\underline{k}, \omega)$ for the Coulomb interaction for a wide range of $\omega$, for example, is difficult at intermediate to low electron density, where correlations are important. Such a calculation would be of interest in any situation where one probes the state density arising from tightly bound outer electron states of impurities and/or atoms of a disordered host.

Lewis Research Center,

National Aeronautics and Space Administration, Cleveland, Ohio, April 28, 1970, 129-03.

\section{REFERENCES}

1. Wolff, P. A.: Theory of the Band Structure of Very Degenerate Semiconductors. Phys. Rev., vol. 126, no. 2, Apr. 15, 1962, pp. 405-412.

2. Langer, J. S.: Theory of Impurity Resistance in Metals. Phys. Rev., vol. 120, no. 3, Nov. 1, 1960, pp. 714-725.

3. Langer, J. S.: Theory of Impurity Resistance in Metals. II. Phys. Rev., vol. 124, no. 4, Nov. 15, 1961, pp. 1003-1010.

4. Langer, J. S.: Evaluation of Kubo's Formula for the Impurity Resistance of an Interacting Electron Gas. Phys. Rev., vol. 127, no. 1, July 1, 1962, pp. 5-16.

5. Langer, J. S.: Thermal Conductivity of a System of Interacting Electrons. Phys. Rev., vol. 128, no. 1, Oct. 1, 1962, pp. 110-117.

6. Betbeder-Matibet, O.; and Nozières, P.: Transport Equation for Quasiparticles in a System of Interacting Fermions Colliding on Dilute Impurities. Ann. Phys. (N. Y.), vol. 37 , no. 1, March, 1966, pp. 17-54.

7. Sigel, James L. ; and Argyres, Petros N.: Transport Equation for a Fermi Liquid in Random Scattering Centers. I. A Quasiparticle Description in the Macroscopic and Low-Temperature Limit. Phys. Rev., vol. 178, no. 3, Feb. 15, 1969, pp. 1016 1036.

8. Sigel, James L.: Derivation of a Quasiparticle Transport Equation for an Impure Fermi Liquid at Low Temperatures. Phys. Rev., vol. 186, no. 1, Oct. 5, 1969, pp. $182-203$.

9. des Cloizeaux, Jacques: Impurity Bands and Perturbation Theory. Phys. Rev., vol. 139A, no. 5, Aug. 30, 1965, pp. 1531-1535. 
10. Pines, David: The Many-Body Problem. W. A. Benjamin, Inc., 1962.

11. Nozières, Philippe: Theory of Interacting Fermi Systems. W. A. Benjamin, Inc., 1964. 
National Aeronautics and Space Administration

WASHINGTON, D. C. 20546

OFFICIAL BUSINESS

FIRST CLASS MAIL

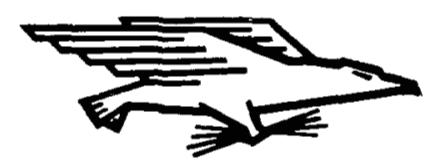

POSTAGE AND FEES PAID NATIONAL AERONAUTICS AND SPACE ADMINISTRATION

$\begin{array}{lllll}04 U & 00151 \quad 51 & 30 S & 70286 & 00903\end{array}$

AIR FORCE WEAPONS LABORATORY /WLOL/

KIRTLAND AFB, NEW MEXICO 87117

ATT E. LOU BOWMAN, CHIEF, TECH. LIBRARY

"The aeronatical and space activities of the United States shall be conducted so as to contribute ... to the expansion of buman knowledge of phenomena in the atmosphere and space. The Administration shall provide for the widest practicable and appropriate dissemination of information concerning its activities and the results thereof."

- National Aeronautics and Space ACt of 1958

\section{NASA SCIENTIFIC AND TECHNICAL PUBLICATIONS}

TECHNICAL REPORTS: Scientific and technical information considered important, complete, and a lasting contribution to existing knowledge.

TECHNICAL NOTES: Information less broad in scope but nevertheless of importance as a contribution to existing knowledge.

TECHNICAL MEMORANDUMS:

Information receiving limited distribution because of preliminary data, security classification, or other reasons.

CONTRACTOR REPORTS: Scientific and technical information generated under a NASA contract or grant and considered an important contribution to existing knowledge.
TECHNICAL TRANSLATIONS: Information published in a foreign language considered to merit NASA distribution in English.

SPECIAL PUBLICATIONS: Information derived from or of value to NASA activities. Publications include conference proceedings, monographs, data compilations, handbooks, sourcebooks, and special bibliographies.

\section{TECHNOLOGY UTILIZATION}

PUBLICATIONS: Information on technology used by NASA that may be of particular interest in commercial and other non-aerospace applications. Publications include Tech Briefs, Technology Utilization Reports and Notes, and Technology Surveys.

Details on the availability of these publications may be obtained from: 